BARber, M. (1955). J. gen. Microbiol. 13, 338-345

\title{
Pigment Production by Staphylococci
}

\author{
BY MARY BARBER \\ Department of Bacteriology, St Thomas's Hospital Medical School, London
}

SUMMARY: White variants, which were stable over many generations, were isolated from six of eight strains of Staphylococcus aureus by cultivation in fluid medium; their incidence was favoured by $0.5 \%(\mathrm{w} / \mathrm{v})$ lithium chloride. These variants all resembled the parent culture from which they were derived in the production of free coagulase and $\alpha$-lysin and, except for one which was non-typable, in bacteriophage type. None of the white variants, however, was clumped by plasma in a slide test, although the parent cultures were.

Golden variants were isolated from two old laboratory strains of coagulase-positive staphylococci which had become white and from three of six white variant cultures isolated in the study of 'aureus $\rightarrow$ albus' variation. These golden variants were isolated under conditions which are unfavourable to the growth of staphylococci in the laboratory. They resembled the parents from which they were derived in production of free coagulase and $\alpha$-lysin and in bacteriophage type. They differed from the parent cultures in production of the factor which causes clumping in plasma, and showed a non-specific increase in resistance to various antibacterial agents.

Although it has long been accepted that pigment production by staphylococci is not a reliable criterion of pathogenicity, nevertheless the majority of strains recently isolated from pathogenic processes do in fact produce a golden pigment. When such strains are repeatedly subcultured in the laboratory they tend to yield white variant colonies, and sometimes the whole culture becomes 'albus'. Chromogenic strains recently isolated from infective processes yield only occasional white variants in the usual laboratory media, but the number can be greatly increased by various means. Pinner \& Voldrich (1932) found that when tuberculous pleural fluid, which causes agglutination of coagulasepositive staphylococci, or an antiserum prepared against a golden strain of staphylococcus was added to broth, eight to ten subcultures were sufficient to convert a golden culture to a pure white culture. Hoffstadt \& Youmans (1932) found a high incidence of white colonies when Staphylococcus aureus strains were serially subcultured in broth containing $0.5 \%(\mathrm{w} / \mathrm{v})$ lithium chloride.

White to gold variation is much less common and indeed only two records of such a change have been found. Pinner \& Voldrich (1932) found that four white strains yielded golden colonies after 20 or more days in undiluted 'antialbus' serum, although over a period of 2 years none had yielded golden colonies spontaneously. Byatt, Jann \& Salle (1948) claimed that a cell-free extract prepared from a highly chromogenic strain of Staphylococcus aureus was capable of inducing chromogenesis in colonies of a white strain of the same species.

The present investigation was undertaken as a result of a chance observation made during other work. Two toxigenic strains of staphylococci were being maintained in mixed culture. Strain $a$ appeared white and was penicillin- 
destroying and streptomycin-sensitive; strain $b$ produced a deep golden pigment and was penicillin-sensitive and streptomycin-resistant. After serial subculture together at various intervals during many months the only variant colonies isolated had the antibiotic sensitivity of strain $a$ but appeared to have the pigment producing capacity of strain $b$. The investigations of Byatt $e t$ al. (1948) appeared to offer a possible explanation for this result. These workers found that when a white strain of Staphylococcus aureus was grown in the presence of a cell-free extract of a golden strain of the same species, a proportion of golden colonies appeared. An extract of my strain $b$ was therefore prepared according to the method of these workers and, again following their technique, my strain $a$ was subcultured daily for $\mathbf{5}$ days in broth + extract of strain $b$; each day the broth cultures of strain $a$ were plated on nutrient agar. The results obtained were similar to those of Byatt, et al. (1948); in repeated experiments different, but often very high, proportions of colonies were deeply pigmented. Since in the process of extraction chloroform, amyl alcohol and ethanol were used, strain $a$ was also subcultured in broth containing small quantities of these substances, but without extract of strain $b$. When subcultures were carried out in the presence of chloroform or ethanol golden colonies appeared, and the incidence of these after subculture in the presence of chloroform was comparable to that obtained with extracts of the chromogenic strain. It seemed clear from these observations that Byatt et al. (1948) were unjustified in concluding that their results were due to a transforming principle extracted from a chromogenic strain, and a more detailed study of pigment variation in strains of staphylococci seemed desirable.

\section{METHODS}

\section{'Aureus $\rightarrow$ albus' variation}

Strains. Eight strains of Staphylococcus aureus yielding typical golden colonies on nutrient agar plates were selected; seven had been recently isolated; six (strains: W 54/36, 37, 38, 39, 40, Rex) from infective processes and one (W 54/129) from a nasal carrier; the remaining culture (V.P. 1) appeared to be a laboratory contaminant. All gave strongly positive reactions in coagulase and $\alpha$-lysin tests, and all produced penicillinase except Rex which was penicillin-sensitive. When tested with bacteriophage, three strains (W 55/ $36,40,129$ ) were found to belong to group I, one strain (V.P. 1) to group II, three strains (W 54/37, 38, Rex) to group III, and one strain (W 55/39) was non-typable.

Cultural procedures. The strains were serially subcultured in Lemco broth (1) alone at $\mathrm{pH} 7 \cdot 4 ;(2)+0.5 \%(\mathrm{w} / \mathrm{v})$ lithium chloride at $\mathrm{pH} 7 \cdot 8$ (as recommended by Hoffstadt \& Youmans, 1932); (3) $+4 \%(\mathrm{v} / \mathrm{v})$ staphylococcal antitoxin (Burroughs Wellcome and Co.). At intervals the broth cultures were plated on Lemco agar and Chapman's (1946) glucose + mannitol + salt medium. These plates were examined after incubation for $18-24 \mathrm{hr}$. at $37^{\circ}$ and again after several days at room temperature. 


\section{'Albus $\rightarrow$ aureus' variation}

Strains. Most of the experiments were carried out with two old laboratory strains which in the course of time had ceased to yield golden colonies under the usual cultural conditions. One of these, strain $\mathrm{D} \mathrm{3}$, is a penicillin-destroying organism of phage group III, isolated in 1948 from the air of a maternity department. The other, NCTC 6571 (Oxford staphylococcus), is a penicillinsensitive organism of phage group I and has an even longer laboratory history. Both have retained their ability to clot plasma and to produce $\alpha$-lysin, although strain NCTC 6571 clots plasma slowly and yields only traces of $\alpha$-lysin. Neither is clumped by plasma. Experiments were also carried out with six of the white variants isolated in the study of 'aureus $\rightarrow$ albus' variation (37 LCalb, 37 Salb, 38 LCalb, 39 Bralb, 129 LCalb, V.P. 1 alb) and with three coagulase-negative strains kindly supplied by the late Professor F. R. Selbie (S 233, 237, 241).

Cultural procedures. Following the preliminary observations with broth containing small quantities of chloroform, various procedures were adopted which created conditions unfavourable for the growth of staphylococci. These included serial subculture in Lemco broth $+2-4 \%(v / v)$ chloroform or staphylococcal antiserum; growth in the presence of sublethal concentrations of penicillin or streptomycin; 3-6 weeks incubation in nutrient broth. As in the study of 'aureus $\rightarrow$ albus' variation, the fluid cultures were plated out at intervals on Lemco agar and Chapman's medium.

Controls of technique. In all procedures involving serial subculture in fluid medium, uninoculated medium was similarly transferred to fresh tubes to test for the likelihood of contaminants. In over 100 such control subcultures one or two contaminating colonies were present on the plates in four instances, but in only one instance was the fluid medium contaminated, so that in the other three, further plating of the broth was negative. Variant colonies, whenever they appeared, were tested for coagulase production, $\alpha$-haemolysin, sensitivity to penicillin and streptomycin and phage-type, to see whether they resembled the parent culture in these tests.

Bacteriophage typing. As a routine, variant cultures were tested only with four phages ( $3 \mathrm{~A}, 3 \mathrm{C}, 47,52 \mathrm{~A})$ since all the parent cultures were sensitive to one or other of these; the method used was that of Williams \& Rippon (1952). The phage-types of a proportion of cultures were kindly confirmed by Dr R. E. O. Williams of the Central Public Health laboratory, who used the full range of phages.

\section{RESULTS \\ 'Aureus $\rightarrow$ albus' variation}

Growth in broth. Although all strains yielded colonies which varied considerably in depth of pigmentation, white colonies were only isolated from two of the seven recently isolated strains $(55 / 39,129)$ and only occasionally from these. Strain V.P. 1 was much more unstable and yielded from 3 to $90 \%$ of white colonies after various periods of subcultivation in broth, although such colonies were rare after surface cultivation on agar. 
Growth in broth + lithium chloride. After 1 or more weeks, with or without subculture in broth containing $0.5 \%$ (w/v) lithium chloride, plate cultures showed colonies which varied very greatly in size and pigmentation. The size ranged from pin-point to $1 \mathrm{~mm}$. or more in diameter and, while some colonies showed a deep golden pigment, some were biscuit-coloured, some white, and some semi-transparent. White colonies were isolated from five of the eight strains: four of the five (W 55/36, 37, 38, 129) yielded occasional white colonies after seven subcultures at 3-day intervals; and the fifth strain (V.P. 1) yielded $90 \%$ after three subcultures. Rough colonies of the type described by Hoffstadt \& Youmans (1932) were not seen.

Growth in broth + antitoxin. Colonies showing moderate variations in size and pigmentation were seen after 1 or more weeks in broth $+4 \%(v / v)$ staphylococcal antitoxic serum, especially when not subcultured. One strain (W 55/ 37) yielded a few white colonies after 3 weeks.

The white variants. White variants were isolated from six of the eight chromogenic strains of Staphylococcus aureus. Plate cultures prepared from them consisted entirely of white colonies, and this remained so after two or three subcultures. These variants retained their ability to clot plasma and to produce $\alpha$-lysin. None of them, however, was clumped by plasma in a slide test, although golden colonies from the same plates and the parent cultures gave positive slide reactions. The white variants isolated from strains $W 55 / 37$, $38,39,129$, and V.P. 1 were of the same phage-type as the parent cultures from which they were derived. The white variant isolated from strain $W 55 / 36$ in lithium chloride broth was non-typable. In no instance was a white variant found to belong to a phage-group different from that of the parent culture.

\section{'Albus $\rightarrow$ aureus' variation}

Strain NCTC 6571. This white strain yielded pigmented variants more readily than any of the other cultures studied. In six experiments this strain was subcultured daily in broth alone or in broth +2 to $4 \%(\mathrm{v} / \mathrm{v})$ chloroform, and in four of these experiments golden colonies were obtained in increasing and often very large numbers from the chloroform-containing cultures. The proportions $(\%)$ of golden colonies on each day in a typical experiment were as follows:

\begin{tabular}{lcccccccrr}
\multicolumn{1}{c}{ Day $\ldots$} & 1 & 2 & 3 & 4 & 5 & 6 & 7 \\
& & & $\%$ & golden colonies \\
In broth & & 0 & 0 & 0 & 0 & 0 & 0 & 0 \\
In broth + chloroform & 0 & 1 & 10 & 8 & 20 & 60 & 90
\end{tabular}

In two of these experiments pigmented colonies were obtained from the broth cultures, but in relatively small numbers. Transparent and semitransparent colonies were sometimes isolated from the chloroform-containing broths.

Strain NCTC 6571 was next serially subcultured on streptomycin ditchplates and a streptomycin-resistant colony selected for further tests. This 
colony, although it gave a pure growth of white colonies on the first few subcultures, proved even more unstable than the parent streptomycin-sensitive culture. Pigmented colonies were obtained when very young $(1 \mathrm{hr}$.) or very old ( 3 or more days), broth cultures were plated and, as with the parent strain, the frequency of occurrence of these colonies was greatly increased by serial subculture in the presence of chloroform.

Further, white streptomycin-resistant colonies selected from NCTC 6571 were rather more stable in relation to pigment, but all yielded golden colonies in the presence of sublethal concentrations of penicillin. This was best demonstrated by the gradient plate technique of Szybalski (1952). On gradient plates with $\mathbf{0 \cdot 1}$ unit penicillin/ml. initially in the upper layer, a zone of pigmented colonies was seen just beyond the minimum concentration of penicillin which caused partial inhibition of growth (Pl. 1, fig. 1).

Strain D3. Studies were made of the parent culture of strain D3 which is penicillin-resistant and streptomycin-sensitive and also of variants which had the following reactions: (1) penicillin- and streptomycin-resistant; (2) penicillin- and streptomycin-sensitive; (3) penicillin-sensitive and streptomycinresistant. These cultures were studied after growth in broth containing chloroform or staphylococcal antiserum, after prolonged incubation in broth, and on penicillin gradient plates. Golden colonies were only isolated from the original penicillin-resistant and streptomycin-sensitive culture and from this only occasionally. Twice golden colonies were isolated from 4- to 6-week-old broth cultures, and once they appeared in increasing number on serial subculture in chloroform broth. No golden colonies were isolated, however, in ten other experiments with chloroform. Transparent or semitransparent colonies were isolated from all of the four kinds of variant when serially subcultured in chloroform broth.

White variants. Golden colonies were isolated from three of the six white variant cultures studied (39 Bralb, 129 LCalb, V.P. 1 alb). In each case the pigmented colonies were obtained when broth cultures which had been incubated at $37^{\circ}$ for 6 weeks were plated out.

Coagulase-negative strains. No golden colonies were isolated from these strains, although search was made for them after serial subculture in chloroform broth, after incubation in broth for many weeks and on penicillin gradient plates. Strain S 241, however, yielded a large number of transparent and semitransparent colonies; the incidence of these was greatly increased by subculture in chloroform broth (Pl. 1, fig. 2).

Golden variants. The golden variants showed a gross difference in pigmentation from the parent strains from which they were isoloted. When first isolated, plate cultures consisted entirely of golden colonies, but all of the variants tended to yield white colonies on further subculture. Streptomycin-resistant pigmented variants of NCTC 6571 were particularly unstable and golden colonies surrounded by satellite white colonies were sometimes seen.

All the pigmented variants showed the same pattern of antibiotic sensitivity as the parent from which they were derived, although those isolated from antibiotic-sensitive parent cultures were frequently able to grow in slightly 
higher concentrations of these antibiotics. Those who have studied the antibiotic sensitivity of staphylococci on solid media will be familiar with the frequent intense pigmentation which occurs just before the inhibitory concentration is reached. This phenomenon differs from that described above, in that on subculture of the deeply pigmented zone in the absence of antibiotic, colonies of the same pigmentation as the parent culture are obtained once more.

The golden cultures showed free coagulase and $\alpha$-lysin production similar to that of the parents from which they were derived, and all here recorded were of the same phage-type as their parent cultures. Unlike the parent cultures most, but not quite all, of the golden variants were clumped by plasma in slide tests. In preliminary experiments golden colonies of phagetype $3 \mathrm{~B} / 3 \mathrm{C} / 55 / 7$ were twice isolated from strain D3 which is of phage-type $6 / 7 / 47 / 53 / 54 / 75+$. These variants have been excluded from the analysis.

The golden variant colonies were frequently, but not always, smaller than the white colonies (cf. Pl. 1, fig. 3). When this was the case growth curves in broth showed that the pigmented culture had a longer lag phase than that of the parent, although after $24 \mathrm{hr}$. of incubation both cultures had multiplied to a similar degree.

Golden variation was associated with a slight but definite increase in resistance to various agents (e.g. chloroform, phenol, penicillin, streptomycin), whereas parent type cultures grew more readily in the usual laboratory conditions. This was best demonstrated by serial subculture of a mixture of organisms in the presence and absence of the antibacterial agent. Thus when an inoculum containing a proportion of one golden variant to 100 white (parent-type) organisms was used and serial transfer of the mixed culture made in broth or in broth $+4 \%(\mathrm{v} / \mathrm{v})$ chloroform, the proportion $(\%)$ of golden colonies was as follows:

\begin{tabular}{|c|c|c|c|}
\hline \multirow{2}{*}{ Day $\ldots$} & \multirow{2}{*}{\multicolumn{3}{|c|}{$\begin{array}{c}2 \\
\% \text { golden colonies }\end{array}$}} \\
\hline & & & \\
\hline brat & $<1$ & $<1$ & 0 \\
\hline loroform culture & 4 & 10 & 25 \\
\hline
\end{tabular}

Thus the golden variants disappeared in broth, whereas they daily increased in numbers in broth + chloroform.

Some of the golden variant cultures survived heating to $60^{\circ}$ for $1 \mathrm{hr}$., although the parent cultures did not survive this treatment. This property, however, was not limited to pigmented variants, as some white colonies isolated in the presence of chlorofrom or antibiotics showed a similar increase in resistance to heat.

\section{DISCUSSION}

It is well known that strains of Staphylococcus aureus when cultivated in vitro tend to lose their capacity to produce a golden pigment. This loss, which is usually first seen in only a proportion of the organisms of the culture, is sometimes associated with a diminished ability to produce $\alpha$-haemolysin and to 
clot plasma. In the present investigation white colonies were only isolated occasionally from any of the seven recently isolated strains of Staphylococcus aurues and not at all from two of them. They were never isolated from surface cultures on agar, but only after subculture in fluid medium. Their isolation was favoured by the presence of $0.5 \%$ (w/v) lithium chloride (cf. Hoffstadt \& Youmans, 1932).

The white variants appeared to resemble organisms of the parent culture in all other properties except that they were no longer clumped by plasma. It has been shown by Duthie (1954) that staphylococci produce two forms of coagulase, one of which is bound to the cell wall and causes clumping in plasma, while the other is free and clots the plasma. The loss by these strains of the clumping factor without loss of the power to clot plasma supports this view.

The 'albus $\rightarrow$ aureus' transformation is not a generally accepted phenomenon, although Pinner \& Voldrich as long ago as 1932 described its occurrence when four white variants of golden strains were cultivated for long periods in undiluted homologous antiserum. The observations of Byatt et al. (1948) are also relevant although, as indicated above, their conclusions seem improbable in the light of the work recorded here.

It is clear from the present work that certain apparently white strains of coagulase-positive staphylococci may yield golden colonies under a variety of conditions which are unfavourable to growth. The most likely explanation of these results is that these golden variants are naturally occurring mutants which, since they show a relative increase in resistance to various antibacterial agents, tend to be selected by them. In this case white variants of golden cultures must also be mutants. That these are much more common is in accordance with current genetical views, since the forward mutation (or loss of a property) is usually much more frequent than the backward mutation (or gain of a property).

Hoffstadt \& Youmans (1932) isolated eight 'rough forms' as well as small colony or G type variants from a single strain of Staphylococcus aureus. When this strain was cultivated in lithium chloride broth the following succession of changes was recorded: smooth yellow (aureus), smooth white, rough white, pseudo rough white, unstable $\mathrm{G}$ form, relatively stable $\mathrm{G}$ form, which, however, tended to revert to the smooth yellow or white form. Coagulase and haemolysin tests were not done, but only the smooth yellow cultures were virulent for rabbits. Similar dissociated forms were isolated from blood cultures of rabbits which had been inoculated with the same strain of $S$. aureus, but under these conditions $\mathbf{G}$ forms appeared more rapidly. The $\mathbf{G}$ forms passed through a Berkefeld filter and showed an increase in thermal death point from 55 to $75^{\circ}$. In their discussion the authors suggest that these dissociated forms are more in the nature of cyclostages than mutants or variants.

In the present work it was found that cultivation in lithium chloride broth led to marked variation in colony size and relatively stable tiny colony variants resembling $\mathbf{G}$ forms were sometimes isolated. Colonies resembling the rough forms of Hoffstadt \& Youmans were not observed, although I have occasionally seen such colonies in old laboratory cultures of staphylococci. 
Journal of General Microbiology, Vol. 13, No. 2

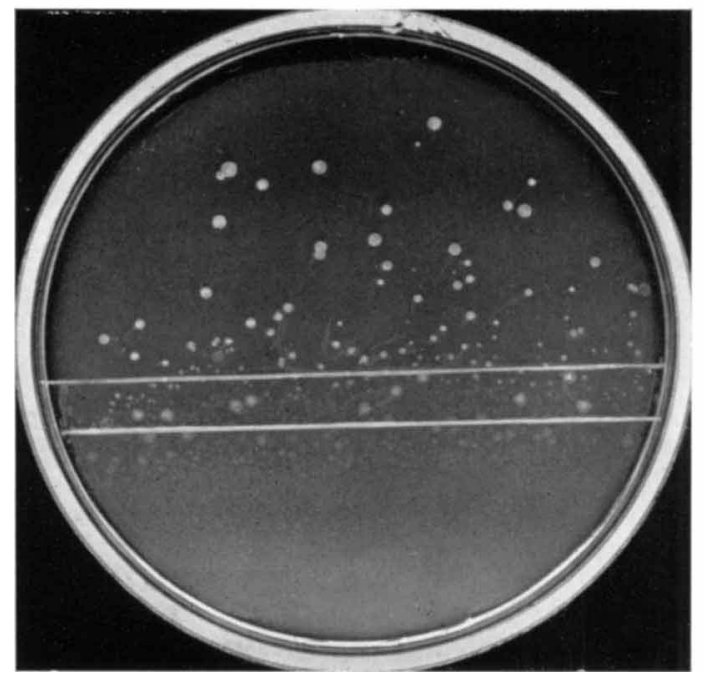

Fig. 1

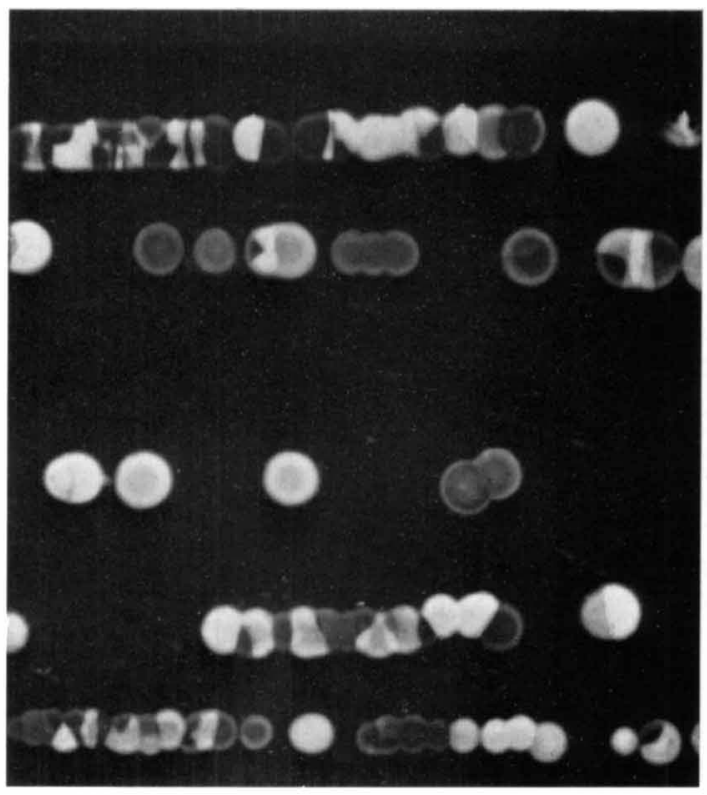

Fig. 2

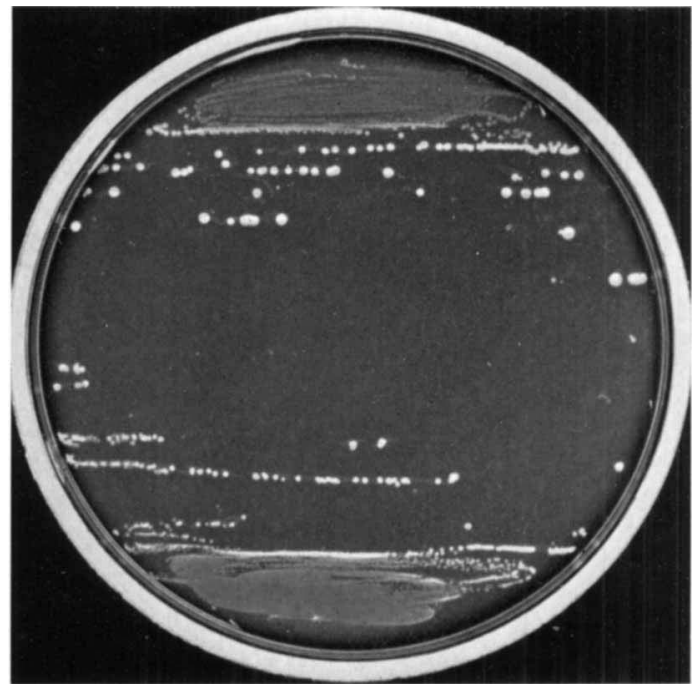

Fig. 3 
Since loss of production of a golden pigment is often associated with diminution or loss of other properties it is tempting to suggest that, under the usual laboratory conditions, a series of mutations takes place until a fully virulent strain of Staphylococcus aureus becomes an avirulent, non-toxigenic, white or even transparent staphylococcus. If this be the case, staphylococci should, perhaps, be regarded as a single species, of which the white coagulase-negative variety is but the laboratory end-product of golden coagulase-positive organisms. Pinner \& Voldrich (1932) even suggested that the difference between 'aureus' and 'albus' cultures is of the same nature as the smooth to rough variation of other micro-organisms. In any case since pigment production is such a variable character it is doubtful whether this should be perpetuated as a basis for classification by use of the terms Staphylococcus aureus and $S$. albus.

My thanks are due to Dr R. E. O. Williams for confirming the bacteriophage type of many of the cultures, and to Mr A. L. Wooding for the photographs.

\section{REFERENCES}

Byatt, P. H., Jann, G. J. \& Salle, A. J. (1948). Variation in pigment production in Staphylococcus aureus. J. Bact. 55, 787.

Chapman, G. H. (1946). The staphylococci. Trans. N.Y. Acad. Sci. 9, 52.

Duthie, E. S. (1954). Evidence for two forms of staphylococcal coagulase. J. gen. Microbiol. 10, 427.

Hoffstadt, R. E. \& Youmans, G. P. (1932). Staphylococcus aureus. Dissociation and its relation to infection and to immunity. J. infect. Dis. 51, 216.

Pinner, M. \& Voldrich, M. (1932). Derivation of Staphylococcus albus, citreus and roseus from Staphylococcus aureus. J. infect. Dis. 50, 185.

Szybalski, W. (1952). Gradient plate technique for study of bacterial resistance. Science, 116, 46.

Williams, R. E. O. \& Rippon, J. E. (1952). Bacteriophage typing of Staphylococcus aureus. J. Hyg., Camb. 50, 320.

\section{EXPLANATION OF PLATE}

\section{Plate 1}

Fig. 1. Penicillin gradient plate seeded with NCTC 6571 (Oxford staphylococcus). Large colonies between the two white lines were golden.

Fig. 2. Strain S 241 plated on nutrient agar after passage in chloroform broth showing large numbers of transparent colonies.

Fig. 3. Strain NCTC 6571 parent culture (upper half) and aureus variant (lower half) plated out on nutrient agar. Note smaller size of colonies of aureus variant. 